\title{
Cor triatriatum dexter with imperforate Ebstein's anomaly
}

\author{
Leon M. Gerlis and Robert H. Anderson ${ }^{1}$ \\ From the Department of Pathology, Grimsby General Hospital; and Department of Paediatrics, Cardiothoracic \\ Institute, Brompton Hospital, London
}

A case of cor triatriatum dexter is described in which the anomalous right atrial partition is identified as the right venous valve; there is an associated anomaly of the right atrioventricular valve which combines features of Ebstein's anomaly, and an atypical, imperforate, tricuspid atresia.

Subdivision of the right atrial cavity into two chambers is a rare cardiac anomaly; to the best of our knowledge only 16 cases have been described previously.

Doucette and Knoblich (1963) reviewed 8 published cases and added a further example; Kauffman and Andersen (1963) found reference to 2 additional reported cases and also added a further personal case; and Jones and Niles (1968) and Runcie (1968) each reported single cases. The condition is generally identified at necropsy but a clinically diagnosed case was described by Verel, Pilcher, and Hynes (1970) and a successful surgical correction following clinical diagnosis was reported by Hansing, Young, and Rowe (1972).

There is an analogous condition in which the dividing membrane is situated very low in the atrium and presentation is that of a supravalvar tricuspid stenosis; Folger (1968) reported 2 such cases and Nakano and his colleagues (1974) described a surgically corrected case.

This malformation should not be confused with a supernumerary right-sided venous chamber caused by dilatation of either the coronary sinus or an anomalous pulmonary vein, as these structures are not parts of the right atrium proper.

The case presently described is of further interest in that the right ventricle is divided by a unique malformation of the tricuspid valve.

${ }^{1}$ R.H.A. is a British Heart Foundation Senior Research Fellow.

\section{Case report}

No clinical history is available; the specimen is known to be from a female child (J.C.); from the size of the heart she was probably between 3 and 6 months old.

Externally the atria are not remarkable, but internally the right atrium is divided in a sagittal plane so as to produce the appearance of three atrial cavities lying side by side. The right lateral compartment (Fig. IA) consists of the trabeculated portion of the atrium and the auricular appendix. The only direct venous inflow to this compartment is from the normally situated coronary sinus. The cavity opens directly into the blind inlet portion of the anomalous right ventricle and the only egress is through a small opening just above the orifice of the coronary sinus which communicates with the medial portion of the divided right atrium.

The medial right atrial compartment is approximately $1.5 \mathrm{~cm}$ in diameter and extends the full length and depth of the atrial portion of the heart. It receives the superior vena cava and the inferior vena cava, both of which are normally situated and it communicates inferiorly with the right lateral atrial compartment. The left wall of this middle atrial cavity is deficient, permitting communication through a wide $0.8 \mathrm{~cm}$ diameter opening with the morphological left atrium. The presence of the limbus fossa ovale enables this connexion to be identified as an ostium secundum defect. The right wall of the middle atrial chamber is a lax membrane which is attached superiorly to the summit of the atrial wall immediately to the right of the superior vena cava opening. Posteriorly it is attached throughout the length of the crista terminalis and inferiorly it approaches. the sinus septum and the inferior limbus of the interatrial septum, thus enclosing the inferior vena cava. At this lower margin it presents a crescent-shaped border to the 

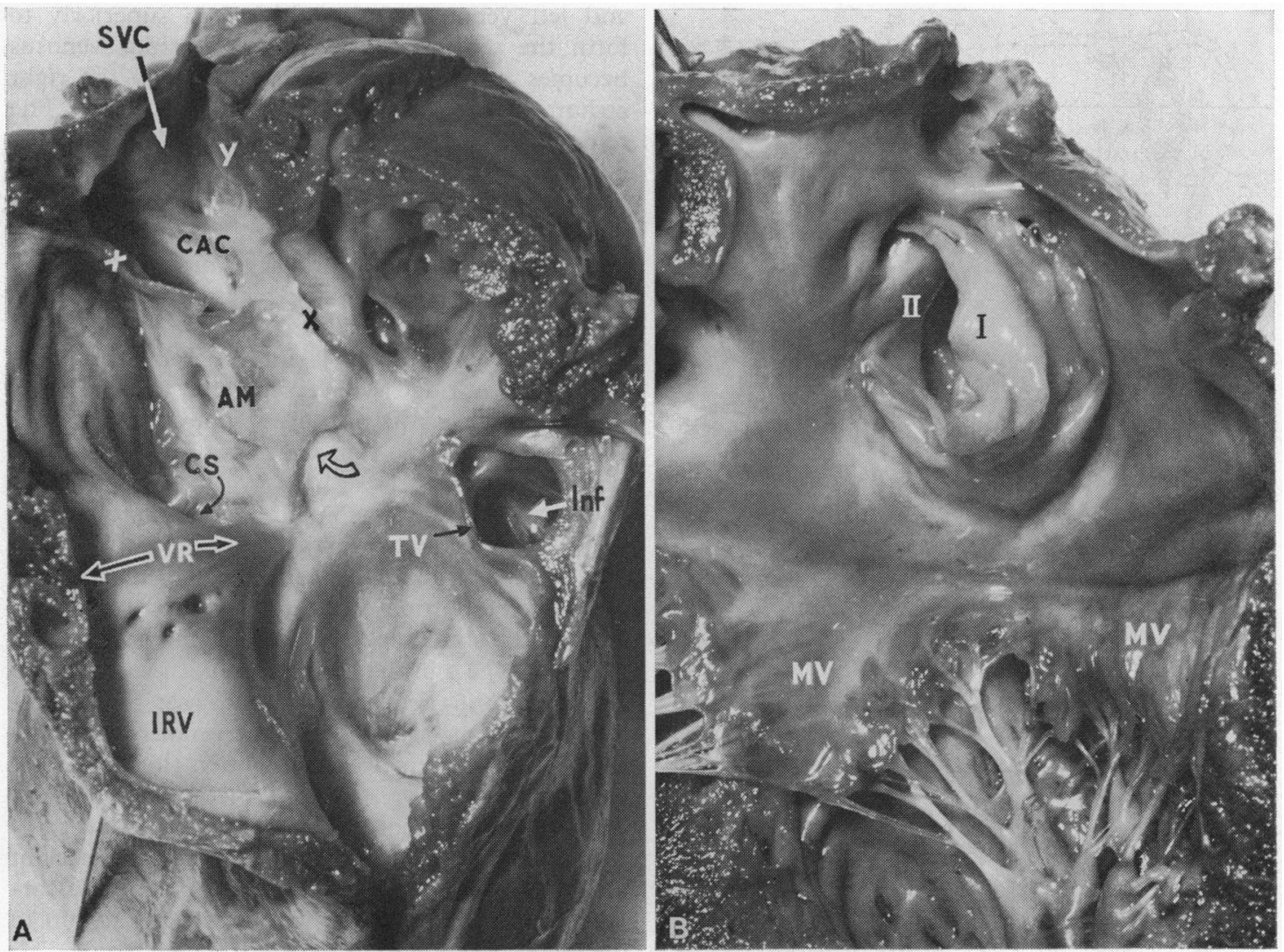

FIG. I $A$ ) The right side of the heart opened laterally showing the division of the right atrium by the atrial membrane $(A M)$, which has been incised in the upper portion along the lines $x x$ and $x y$ to reveal the opening of the superior vena cava $(S V C)$ into the central atrial compartment $(C A C)$. The coronary sinus orifice is indicated (CS) and the adjacent opening in the atrial membrane is marked by the open arrow. The bare valve ring $(V R)$ lies between the right atrium and the inlet portion of the right ventricle (IRV) which has a smooth atrialized wall. The vestigial medial portion of the tricuspid valve (TV) separates the inlet portion from the infundibular portion (inf.); these structures have been opened by the plane of incision, there is no natural communication between the infundular cavity and any other cardiac chamber, neither has it any outlet as the pulmonary valve is atretic. B) The medial wall of the left atrium showing the aneurysmal portion of the septum primum (I) through the opening of which can be seen the smooth margin of the septum secundum (II). The mitral valve (MV) is normally formed.

small $0.25 \mathrm{~cm}$ diameter communication with the right lateral cavity (Fig. IA). Anteriorly the partition is attached to the atrial wall on a line passing downwards from the cleft between the superior vena cava orifice and the opening of the auricular appendix. The posterior wall of this central cavity is smooth and free from muscular trabeculations.

The left atrium is of normal configuration and receives the normal pulmonary veins. The wall between it and the central cavity exhibits the normal features of the left side of the interatrial septum, with an ostium secundum defect. The very lax septum primum portion of the septum bulges aneurysmally into the left atrium (Fig. IB).
The left atrium opens into the normal left ventricle through a normal mitral valve (Fig. IB). The right atrioventricular valve is grossly abnormal; the major portion of the valve ring is smooth and shows no trace of the valve. The entrance into the ventricle is wide and unguarded, and the appearances are those of Ebstein's anomaly with atrialization of most of the lateral and anterior walls of the hypoplastic right ventricular in!et chamber (Fig. IA). On the medial aspect of the valve ring a vestigial valve cusp forms an occluding partition across the right ventricle completely separating the inlet chamber from a diminutive, $1.5 \mathrm{~cm}$ long, $0.5 \mathrm{~cm}$ diameter, outlet chamber which extends to the site of the atretic pulmonary valve. 

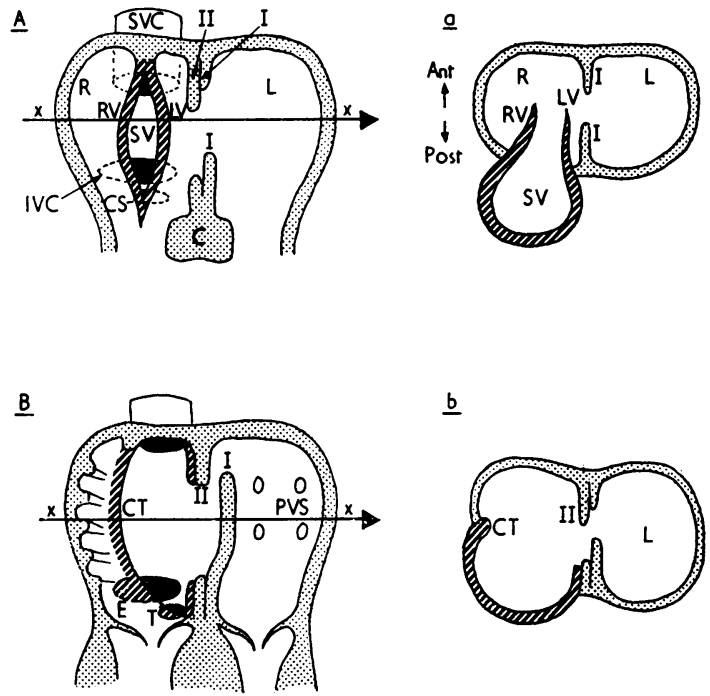

$\underline{b}$
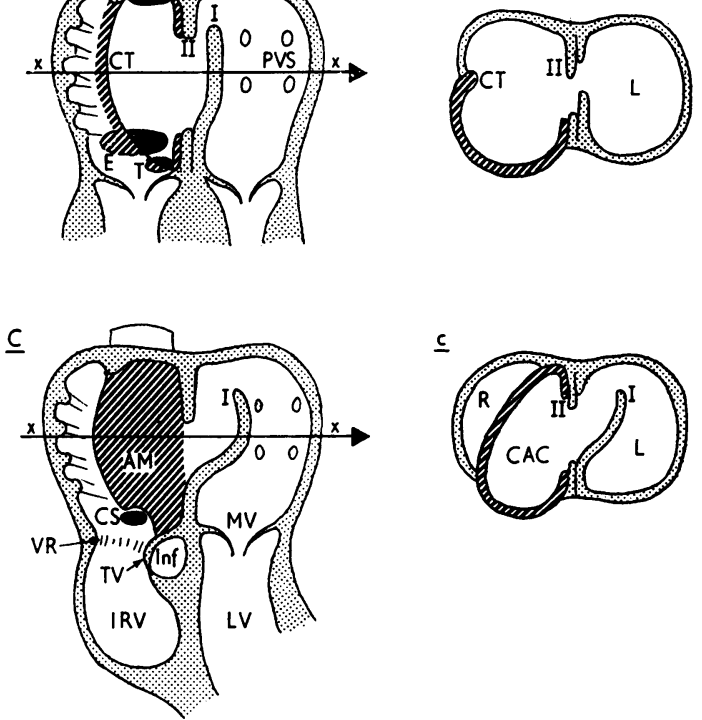

FIG. 2 Diagrams showing the structures involved in atrial septation: $A$. embryonic; $B$. normal mature state; C. Cor triatriatum dexter (present case). The inset diagrams $a, b, c$, represent cross-sectional views in the planes $x x$ as indicated. $R$, right atrium; $L$, left atrium; $S V$, sinus venosus; $R V$, right venous valve; $L V$, left venous valve; $C$, atrioventricular cushion; $C T$, crista terminalis; $E$, Eustachian valve; $T$, Thebesian valve; PVS, pulmonary veins; IVC, inferior vena cava; other symbols as for Fig. I.

The great vessels are in normal relation, the pulmonary artery trunk is atretic, and the ductus arteriosus is patent.

\section{Discussion}

As with other reported cases of cor triatriatum dexter, the right atrial partition is undoubtedly derived from an abnormal and persistent right venous valve. In early stages of cardiac development the opening of the sinus venosus into the right atrium is guarded by two prominent folds, the right and left venous valves, which join superiorly to form the septum spurium. As the sinus venosus becomes progressively incorporated into the right atrium, these valves separate and regress. The remnant of the left venous valve merges with the atrial septum secundum. The right venous valve normally disappears except for the Eustachian and Thebesian valves at the lateral margins of the inferior vena cava and coronary sinus, respectively. These orifices are separated by the inferior limbic band or sinus septum. The site of the remainder of the right venous valve is indicated by the crista terminalis, a ridge which separates the pectinate muscle region of the primitive right atrial chamber from the smooth portion which is derived from the sinus venosus.

The position of attachment of the atrial partition in this case, together with the absence of a separately recognizable Eustachian valve, identifies it as being the portion of the right venous valve superior to the sinus septum. Such an anomaly corresponds to type 2 in the classification of Doucette and Knoblich (1963). In most similar cases the anteroinferior margin of the persistent venous valve presented a prominent free edge, often thickened and cordlike; in the present case there is an unusual degree of attachment to the anterior wall, and communication between the two portions of the right atrium is minimal and provides solely for the coronary venous flow across from the right atrium to the left.

Cases of this anomaly dying during infancy and childhood generally have atresia or stenosis of the pulmonary valve, usually in conjunction with atresia or hypoplasia of the right atrioventricular valve. Coexisting Ebstein's anomaly of the tricuspid valve has also been described (Dubin and Hollingshead, 1944). Though the contention that there is a special association between the two anomalies has been rejected by Hudson (1965), the present case lends support to such a hypothesis.

A remarkable feature of this case is that the tricuspid valve is imperforate in addition to exhibiting Ebstein's anomaly. The valve presents a dense membrane at the junction of the hypoplastic inflow and outflow portions of the right ventricle. Such imperforate Ebstein anomalies are very rare; one of us (R.H.A.) has seen one additional example in association with a ventricular septal defect but the present case is associated with an intact ventricular septum. We are unaware of any previous description of this anomaly. Though this condition can correctly be called tricuspid atresia, it is important to distinguish it from the commoner form of tricuspid atresia. The latter anomaly more closely resembles a state of morphologically single ventricle 
with outlet chamber. Thus, the supposed site of the atretic valve points to the primitive ventricle and not to the 'right' ventricle. In contrast, in the present case the imperforate valve interposes between the inlet and outlet portions of a developed but hypoplastic right ventricle.

\section{References}

Doucette, J., and Knoblich, R. (1963). Persistent right valve of the sinus venosus. Archives of Pathology, 75, 105.

Dubin, I. N., and Hollingshead, W. H. (1944). Congenitally insufficient tricuspid valve accompanied by an anomalous septum in the right atrium. Archives of Pathology, 38, 225.

Folger, G. M. (1968). Supravalvular tricuspid stenosis; association with developmental abnormalities of the right heart and derivatives of the sixth aortic arch. American fournal of Cardiology, 21, 81.

Hansing, C. E., Young, W. P., and Rowe, G. G. (1972). Cor triatriatum dexter; persistent right sinus venosus valve. American fournal of Cardiology, 30, 559.
Hudson, R. E. B. (1965). Cardiovascular Pathology. Arnold, London.

Jones, R. N., and Niles, N. R. (1968). Spinnaker formation of the sinus venosus valve. Circulation, 38, 468.

Kauffman, S. L., and Andersen, D. H. (1963). Persistent venous valves, maldevelopment of the right heart and coronary artery - ventricular communications. American Heart fournal, 66, 664.

Nakano, S., Kawashima, Y., Miyamoto, T., Kitamura, S., and Manabe, H. (1974). Supravalvular tricuspid stenosis resulting from persistent right sinus venosus valve. Annals of Thoracic Surgery, 17, 591.

Runcie, J. (1968). A complicated case of cor triatriatum dexter. British Heart fournal, 30, 729.

Verel, D., Pilcher, J., and Hynes, D. M. (1970). Cor triatrium dexter. British Heart fournal, 32, 714.

Requests for reprints to Dr. L. M. Gerlis, Department of Pathology, Grimsby General Hospital, Grimsby, South Humberside. 